ARTICLE

DOI: $10.1038 / s 41467-018-06600-8$

\title{
High-content ductile coherent nanoprecipitates achieve ultrastrong high-entropy alloys
}

Yao-Jian Liang ${ }^{1}$, Linjing Wang ${ }^{1}$ Y Yuren Wen², Baoyuan Cheng ${ }^{1}$, Qinli Wu ${ }^{3}$, Tangqing Cao ${ }^{1}$, Qian Xiao', Yunfei Xue ${ }^{1}$, Gang Sha ${ }^{3}$, Yandong Wang ${ }^{4}$, Yang Ren ${ }^{5}$, Xiaoyan $\mathrm{Li}^{6}$, Lu Wang ${ }^{1}$, Fuchi Wang ${ }^{1} \&$ Hongnian Cai ${ }^{1}$

Precipitation-hardening high-entropy alloys ( $\mathrm{PH}-\mathrm{HEAs}$ ) with good strength-ductility balances are a promising candidate for advanced structural applications. However, current HEAs emphasize near-equiatomic initial compositions, which limit the increase of intermetallic precipitates that are closely related to the alloy strength. Here we present a strategy to design ultrastrong HEAs with high-content nanoprecipitates by phase separation, which can generate a near-equiatomic matrix in situ while forming strengthening phases, producing a $\mathrm{PH}-\mathrm{HEA}$ regardless of the initial atomic ratio. Accordingly, we develop a non-equiatomic alloy that utilizes spinodal decomposition to create a low-misfit coherent nanostructure combining a near-equiatomic disordered face-centered-cubic (FCC) matrix with high-content ductile $\mathrm{Ni}_{3} \mathrm{Al}$-type ordered nanoprecipitates. We find that this spinodal order-disorder nanostructure contributes to a strength increase of $\sim 1.5 \mathrm{GPa}$ ( $>560 \%$ ) relative to the HEA without precipitation, achieving one of the highest tensile strength (1.9 GPa) among all bulk HEAs reported previously while retaining good ductility (>9\%).

\footnotetext{
${ }^{1}$ School of Materials Science and Engineering, Beijing Institute of Technology, 100081 Beijing, China. ${ }^{2}$ Beijing National Laboratory for Condensed Matter Physics, Institute of Physics, Chinese Academy of Sciences, 100190 Beijing, China. ${ }^{3}$ Herbert Gleiter Institute of Nanoscience, Nanjing University of Science and Technology, 210094 Nanjing, China. ${ }^{4}$ State Key Laboratory for Advanced Metals and Materials, University of Science and Technology Beijing, 100083 Beijing, China. ${ }^{5}$ X-ray Science Division, Argonne National Laboratory, Argonne, IL 60439, USA. ${ }^{6}$ Center for Advanced Mechanics and Materials, Applied Mechanics Laboratory, Department of Engineering Mechanics, Tsinghua University, 100084 Beijing, China. These authors contributed equally: Yao-Jian Liang, Linjing Wang, Yuren Wen. Correspondence and requests for materials should be addressed to Y.X. (email: xueyunfei@bit.edu.cn) or to X.L. (email: xiaoyanlithu@tsinghua.edu.cn) or to F.W. (email: wangfuchi@bit.edu.cn)
} 
$\mathrm{H}$ igh-entropy alloys (HEAs), which contain at least four principal elements in (near-)equiatomic ratios, have attracted extensive attention because of their interesting properties $^{1-6}$. In the early stage of the development of HEAs, researchers wanted to seek single-phase solid-solution alloys because they considered that intermetallics are brittle and may degenerate the properties of HEAs ${ }^{1-4}$. However, the fact that in most engineering alloys, secondary phases contribute significantly to the alloy properties is also true in HEAs; many reported HEAs that can overcome the strength-ductility trade-off contain two or more phases ${ }^{7-17}$. Proper control of the type, shape, size, volume fraction, and distribution of precipitation phases is critical for the development of the HEAs with high strength while retaining sufficient ductility ${ }^{6-8,13-17}$. $\mathrm{Ni}_{3} \mathrm{Al}$-type ordered $\mathrm{Ll}_{2}$ phases, which are ductile and coherent with FCC matrices ${ }^{18}$, are one important class of strengthening phases in FCC-based alloys such as superalloys, and the strengthening effects depend prominently on the volume fractions of these precipitates ${ }^{19-21}$. Similar to conventional FCC alloys, the HEAs strengthened by $\mathrm{L}_{2}$ precipitates also possess good balances between strength and ductility ${ }^{5,13-17}$. However, the current alloying concept of HEAs, which emphasizes near-equiatomic initial compositions, intrinsically limits the increase in the contents of these ductile intermetallic strengthening phases $7,8,13-17$. As schematically shown in Fig. 1a, nearequiatomic HEAs tend to form brittle intermetallics (generally near-equiatomic, e.g. NiAl-type B2 and complex $\sigma$ phases) $)^{7,8,13}$; formation of ductile $\mathrm{L}_{2}$ phases requires a high $\mathrm{Ni} / \mathrm{Al}$ ratio. As a result, inclusion of $\mathrm{Ll}_{2}$ precipitates into FCC HEAs has to decrease the concentration of the $\mathrm{Ll}_{2}$ forming elements to a value $<7$ at $\%$ in order to obtain the near-equiatomic matrices, e.g. $\mathrm{Al}_{0.3} \mathrm{CoCrFeNi}^{13}$ and $(\mathrm{CoCrFeNi})_{94} \mathrm{Ti}_{2} \mathrm{Al}_{4}{ }_{4}{ }^{17}$. In other words, based on the alloying strategy/concept of HEAs, a high Ni/Al ratio that promotes the formation of $\mathrm{Ll}_{2}$ phases can only be achieved by reducing the $\mathrm{L}_{2}$ former because the $\mathrm{Ni}$ additions in near-equiatomic HEAs are commonly $<25$ at $\%$. Such low concentrations of the $\mathrm{Ll}_{2}$ former lead to difficulty in developing stronger HEAs with large $\mathrm{L}_{2}$ volume fractions ${ }^{14-17}$.
To obtain a HEA with high-content $\mathrm{L}_{2}$ strengthening phases, we use a different way to increase the $\mathrm{Ni} / \mathrm{Al}$ ratio by adding more $\mathrm{Ni}$ element rather than reducing $\mathrm{Al}$ concentration. This composition design aims to ensure that the phase separation can generate a near-equiatomic FCC matrix in situ while forming highcontent $\mathrm{L}_{2}$ phases, creating PH-HEAs with superior strength (Fig. 1b). Based on this strategy, we develop a non-equiatomic system $\mathrm{Al}_{0.5} \mathrm{Cr}_{0.9} \mathrm{FeNi}_{2.5} \mathrm{~V}_{0.2}$, which has a high $\mathrm{Ni}$ concentration of $\sim 50$ at $\%$ and a high Ni/Al ratio of $\sim 5$. Moreover, our alloy excludes Co to reduce the cost and contains $\sim 4$ at $\% \mathrm{~V}$ to stabilize and strengthen $\mathrm{Ll}_{2}$ phases ${ }^{20}$. This well-designed non-equiatomic system can form a PH-HEA in situ by utilizing spinodal decomposition to create a low-misfit coherent nanostructure composed of a near-equiatomic disordered FCC matrix and highcontent ordered $\mathrm{L} 1_{2}$ nanoprecipitates $(>50 \mathrm{vol} \%)$. This distinctive spinodal order-disorder nanostructure can resist dislocation motion by providing strong diffuse obstacles and by creating antiphase boundaries (APBs) on the slip planes of these ordered $\mathrm{L1}_{2}$ nanophases ${ }^{19}$, contributing to an ultrahigh strength while retaining good ductility and work-hardening capacity. We anticipate that this alloying strategy can offer more possibilities for the compositional design of HEAs, and will open new avenues for development of ultrastrong and ductile HEAs superior to existing commercial alloys.

\section{Results}

Material properties. Tensile engineering stress-strain curves (Fig. 2a) show that although the solution-treated (ST) sample is soft (yield strength of $\sim 274 \mathrm{MPa}$ ), remarkable strength increases occur in the samples after $72 \%$ cold rolling followed by ageing for $1 \mathrm{~h}$ at $600{ }^{\circ} \mathrm{C}$ and $700{ }^{\circ} \mathrm{C}$ (hereinafter named $600 \mathrm{~A}$ and $700 \mathrm{~A}$, respectively). The maximum strengthening observed in $600 \mathrm{~A}$ is $>1500 \mathrm{MPa}$ (strength increment $>560 \%$ ), achieving ultrahigh yield strength (YS) of $1810 \mathrm{MPa}$ and ultimate tensile strength (UTS) of $1905 \mathrm{MPa}$. Moreover, the YS and UTS of $700 \mathrm{~A}$ are $1570 \mathrm{MPa}$ and $1763 \mathrm{MPa}$, respectively, which are $\sim 1200 \mathrm{MPa}$ a

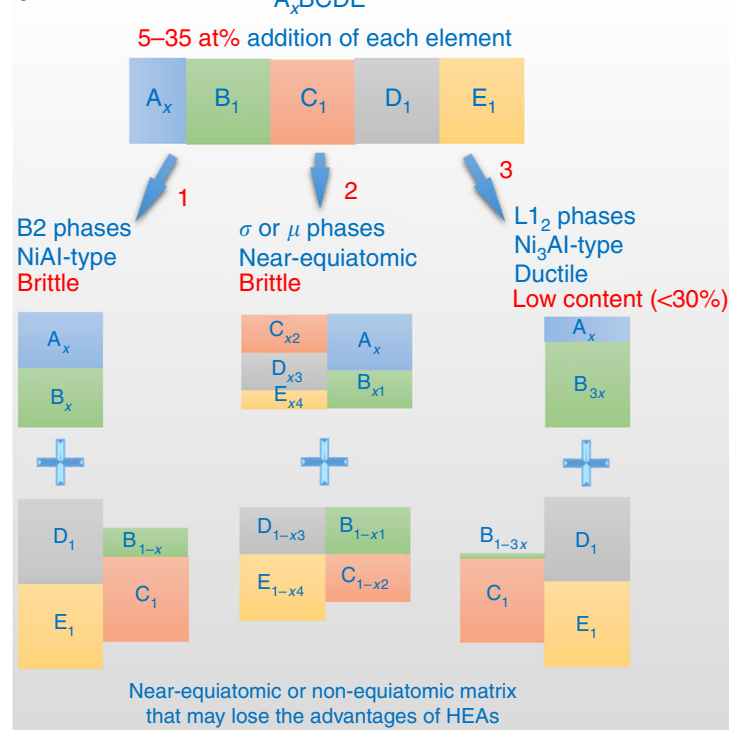

b

$\mathrm{A}_{x} \mathrm{~B}_{1+3 x} \mathrm{CDE}$ Non-equiatomic (B up to 50 at\%)

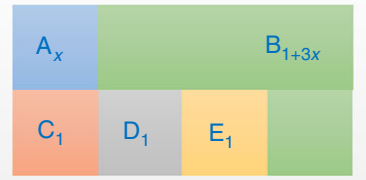

Phase separation

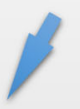

High content (>50\%)

ductile precipitates

e.g. $\mathrm{Ni}_{3} \mathrm{Al}$-type $\mathrm{L1}_{2}$ phases

Near-equiatomic Matrix

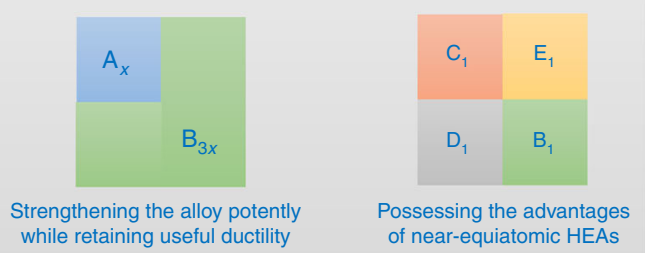

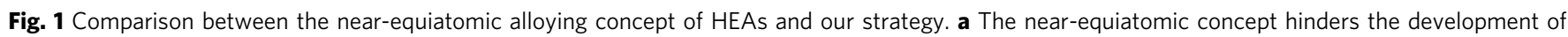

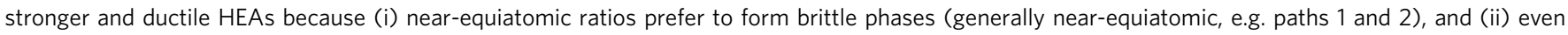
though decreasing the $\mathrm{Al}$ concentrations can increase $\mathrm{Ni} / \mathrm{Al}$ ratios and obtain ductile $\mathrm{L1}_{2}$ phases (path 3 ), near-equiatomic compositions limit the Ni concentrations and hence the $\mathrm{L}_{2}$ phase contents. b Our alloying strategy aims to obtain a final microstructure combining a near-equiatomic matrix with high-content ductile precipitates, regardless of the initial atomic ratio 

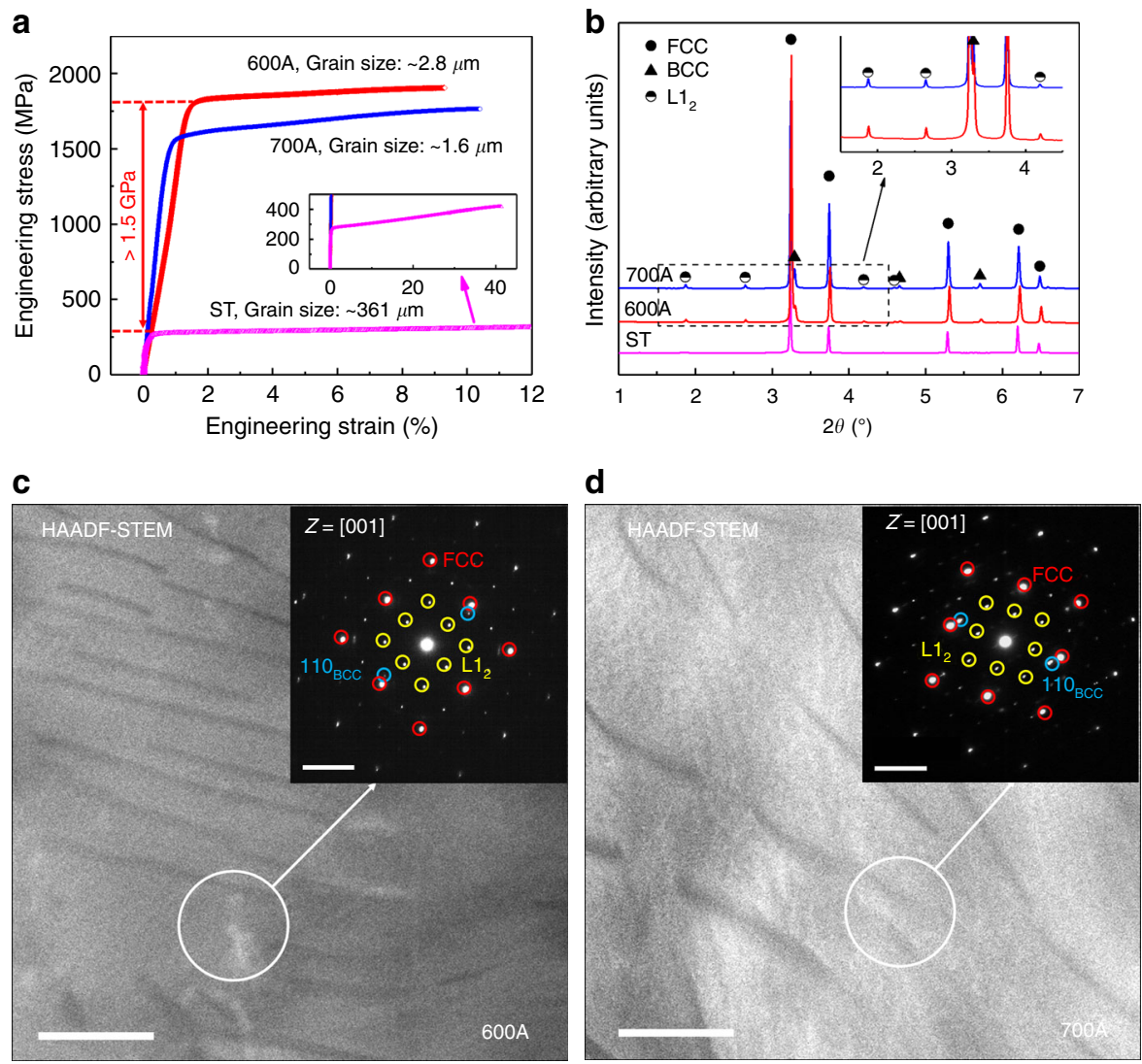

Fig. 2 Mechanical properties and phase constituent of our alloy prepared under various processing conditions. a Tensile engineering stress-strain curves showing remarkable increases in strength occurring after cold-rolling and ageing (>1500 MPa for $600 \mathrm{~A}$ and $\sim 1200 \mathrm{MPa}$ for $700 \mathrm{~A}$ compared with the ST sample). b HEXRD spectra showing that the aged samples contain FCC, $L 1_{2}$, and BCC phases, whereas the ST sample is a single-phase FCC alloy. c, d HAADF-STEM images of $600 \mathrm{~A}$ and $700 \mathrm{~A}$, respectively. Scale bar, $200 \mathrm{~nm}$. The SAED patterns in the insets verify the phase composition in the aged samples. Scale bar, $5 \mathrm{~nm}^{-1}$

( $430 \%)$ higher than those of the ST sample. In addition, both $600 \mathrm{~A}$ and $700 \mathrm{~A}$ exhibit good ductility ( 9-10\%) and excellent work-hardening capacity (uniform elongation to fracture). The exceptional combination of properties makes our alloy $\mathrm{Al}_{0.5} \mathrm{Cr}_{0.9} \mathrm{FeNi}_{2.5} \mathrm{~V}_{0.2}$ superior to most traditional alloys for potential advanced structural applications.

Phase constituent. After cold rolling and ageing, the grains in $600 \mathrm{~A}$ and $700 \mathrm{~A}$ become very fine (Supplementary Fig. 1). However, the strength increases resulting from grain boundaries are only $212 \mathrm{MPa}$ in $600 \mathrm{~A}$ and $284 \mathrm{MPa}$ in $700 \mathrm{~A}$ (see Methods and Supplementary Fig. 2 for calculated details), indicating that the ultrahigh strength of the samples arises from other mechanisms. Synchrotron high-energy X-ray diffraction (HEXRD) spectra of both $600 \mathrm{~A}$ and $700 \mathrm{~A}$ show superlattice peaks of the ordered $\mathrm{L}_{2}$ structure and characteristic peaks of the bodycentered-cubic (BCC) structure, in addition to characteristic peaks of an FCC-structured matrix (Fig. 2b). Quantitative analysis of the volume fraction of each phase based on the HEXRD results reveals that in the two samples, the $\mathrm{L}_{2}$ phases are the major precipitates ( $\sim 50 \%$ in $600 \mathrm{~A}$ and $\sim 44 \%$ in $700 \mathrm{~A})$, whereas the contents of the BCC phases are barely $\sim 6 \%$, confirming the validity of our strategy for designing $\mathrm{PH}$-HEAs with high-content ductile $\mathrm{L}_{2}$ strengthening phases. Interestingly, only two regions (bright matrix and dark precipitates with low volume fractions) are found in high-angle annular dark-field (HAADF) scanning transmission electron microscopy (STEM) images (Fig. 2c, d). However, selected area electron diffraction (SAED) patterns (insets in Fig. 2c, d) indicate that the samples indeed contain three phases-two symmetric spots exist near $\{200\}_{\mathrm{FCC}}$ in addition to the superlattice patterns of the FCC and $\mathrm{L}_{2}$ phases. The interplanar spacings of the two spots are approximately $0.205 \mathrm{~nm}$, which is consistent with the spacings of $\{110\}_{\mathrm{BCC}}$ calculated from the HEXRD spectra $(\sim 0.204 \mathrm{~nm})$, suggesting that these low-content dark precipitates are of BCC structure and that the bright region consists of the FCC matrix and high-content $\mathrm{L}_{2}$ strengthening phases.

Spinodal order-disorder nanostructure. To reveal the microstructure of the FCC matrix and $\mathrm{Ll}_{2}$ phases, we conducted atomic-resolution HAADF-STEM using atomic mass contrast and atom probe tomography (APT). We find an interesting nanostructure consisting of two fully coherent nanoscale regions in atomic-resolution images (Fig. 3a), in which brighter columns represent heavier elements (e.g. Ni) whereas darker columns refer to lighter atoms (e.g. Al). The intensity profile along the cyan arrow across the two regions in Fig. 3a reveals that heavier and lighter elements are arranged alternately in one region, whereas in the other region the intensity is uniform (Fig. 3b). Fast Fourier transform (FFT) of the region with uniform intensity presents an FCC-structured diffraction pattern, whereas that of the alternately arranged region demonstrates an $\mathrm{L1}_{2}$-type superlattice pattern. Interestingly, the two nanophases seem to be tangled and have no abrupt changes in composition (composition continuously changing without abrupt interfaces), and the lattice parameters of the two highly coherent phases are almost the same $(0.3582 \mathrm{~nm}$ for FCC and $0.3583 \mathrm{~nm}$ for $\mathrm{Ll}_{2}$ ). Consequently, these $\mathrm{L}_{2}$ phases are invisible in Fig. $2 c$, d. Hereinafter we mainly present the APT 

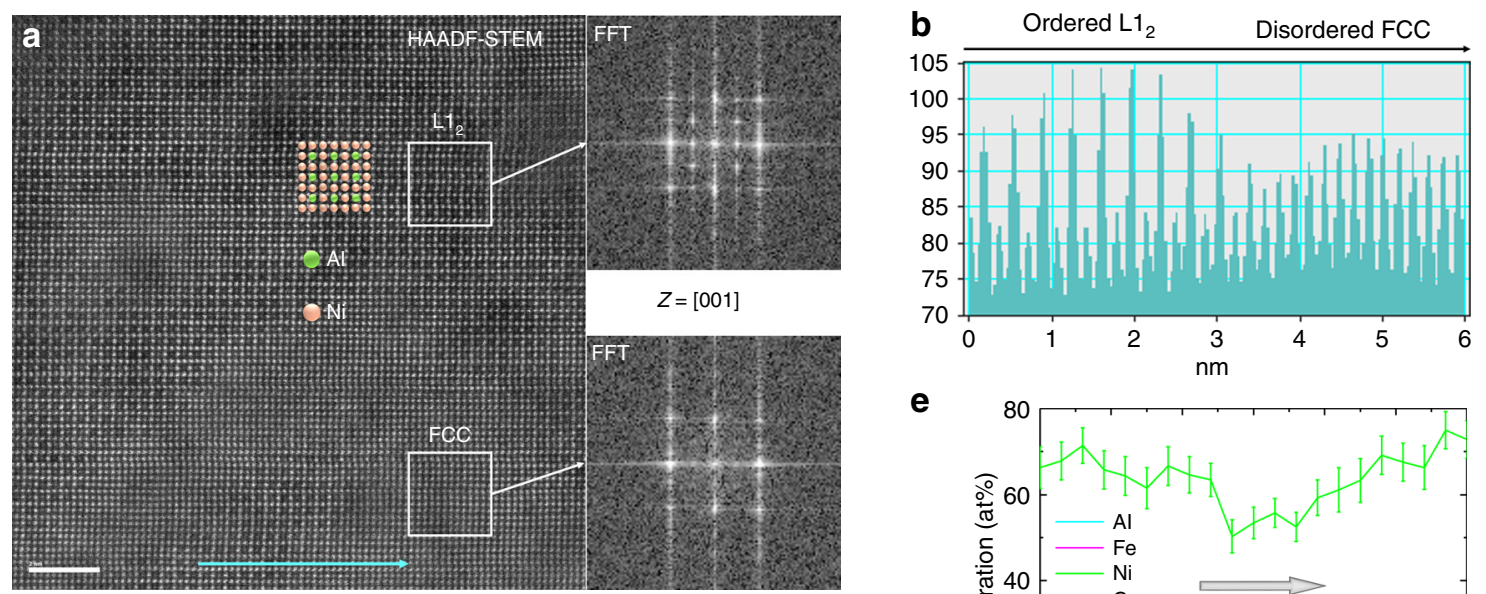

e

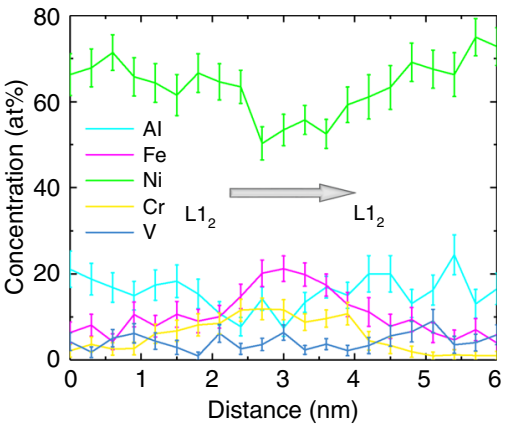

C

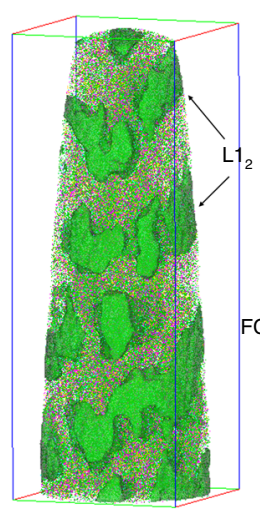

63 at\% $\mathrm{Ni}$ d 15 at\% $\mathrm{Cr}$

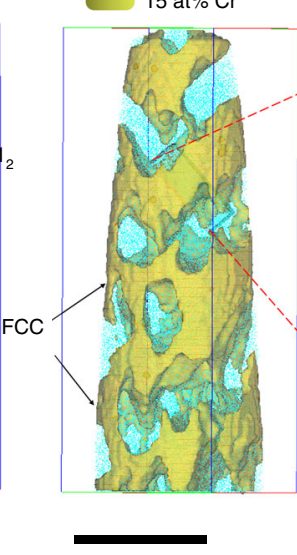

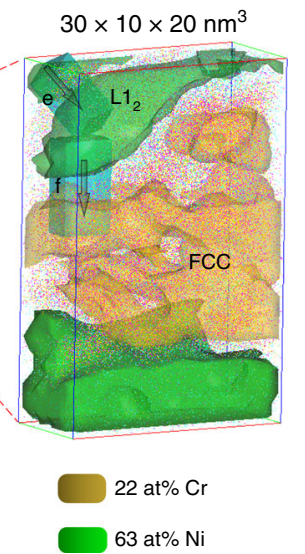

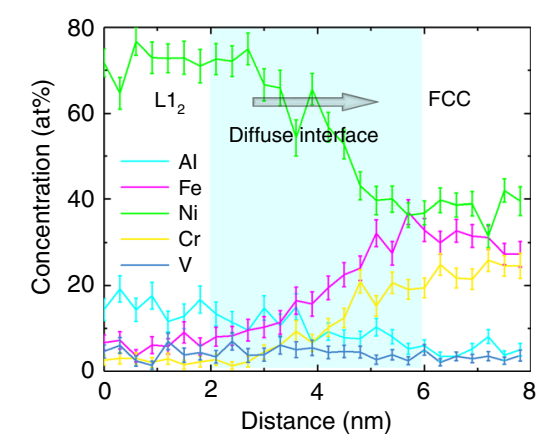

Fig. 3 Composition, morphology and structure of phases existing in the bright matrix region of $600 \mathrm{~A}$. a Atomic mass contrast in atomic-resolution HAADF-STEM images revealing a distinctive nanostructure consisting of a disordered FCC matrix and ordered $\mathrm{Ll}_{2}$ phases with diffuse coherent interfaces. Scale bar, $2 \mathrm{~nm}$. $\mathbf{b}$ Intensity profile along the cyan arrow marked in a showing atomic arrangement in the two phases. c, $\mathbf{d}$ Three-dimensional reconstruction of 63 at $\% \mathrm{Ni}$ and 15 at\% $\mathrm{Cr}$ isoconcentration surfaces presenting the morphologies of the ordered $\mathrm{L}_{2}$ precipitates and disordered FCC matrix, respectively. A box was selected from $d$ to provide more details about the morphology and composition of the two phases. Scale bar, $40 \mathrm{~nm}$. e, $\mathbf{f}$ One-dimensional concentration profiles showing the element distributions from $L 1_{2}$ to $L 1_{2}$ and $L 1_{2}$ to $F C C$, respectively. Error bars, s.d.

results of the detailed microstructure of $600 \mathrm{~A}$ because the microstructures of $600 \mathrm{~A}$ and $700 \mathrm{~A}$ are similar (Supplementary Fig. 3).

The three-dimensional morphologies of the ordered $\mathrm{L}_{2}$ nanoprecipitates and disordered FCC matrix are revealed by reconstructing 63 at $\% \mathrm{Ni}$ and 15 at\% $\mathrm{Cr}$ isoconcentration surfaces, respectively, based on the APT data (Fig. 3c, d). The tangled nanostructure can be seen more clearly, where the interconnected disordered FCC matrix serves as the frame and is filled with the ordered $\mathrm{L}_{2}$ phases (also interconnected). Interestingly, this tangled nanostructure with diffuse coherent precipitate-matrix interfaces seems to be an isotropic spinodal structure $^{22}$. To further investigate the composition and morphology at the phase interfaces, we selected an analysis box of $30 \times$ $20 \times 10 \mathrm{~nm}^{3}$ from Fig. $3 \mathrm{~d}$ and reconstructed an isoconcentration surface with a higher $\mathrm{Cr}$ content (22 at\%) within it. The onedimensional concentration profiles of phase interfaces (Fig. 3e, f) demonstrate that Fe slightly enriches at the $\mathrm{L}_{2}-\mathrm{L}_{2}$ interface and that all elements continuously change from FCC to $\mathrm{L}_{2}$ without an abrupt FCC- $\mathrm{L}_{2}$ interface (diffuse interface width $\sim 4$ $\mathrm{nm}$ ). The one-dimensional concentration profiles across multiple FCC and $\mathrm{L1}_{2}$ phases also exhibit long-range periodic composition fluctuations without abrupt changes in composition
(Supplementary Fig. 4). These results suggest the spinodally decomposed nature of the phase separation ${ }^{22}$. The mean compositions of the two phases confirm that the FCC matrix is a phase conforming to the near-equiatomic alloying concept of HEAs and that our alloying strategy for producing PH-HEAs in situ by phase separation is valid (Supplementary Fig. 4c). Moreover, the APT results indicate that the BCC phases are $\mathrm{Cr}$ rich lamellar phases with the interfaces slightly enriched by $\mathrm{Fe}$ (Supplementary Fig. 5).

\section{Discussion}

To form a near-equiatomic matrix in situ, we add a high content of $\mathrm{Cr}(\sim 18$ at $\%)$ into our alloy. Such a high addition promotes the change of the phase separation mechanism to spinodal decomposition $^{23}$ and reduces the difference in the lattice parameters between the FCC matrix and $\mathrm{L}_{2}$ nanophases ${ }^{24}$, thereby stabilizing the high-density coherent interfaces ${ }^{25}$ and lowering the driving force for competitive coarsening ${ }^{26}$. During phase separation, both $\mathrm{Cr}$ and $\mathrm{Fe}$ are rejected from the $\mathrm{L1}_{2}$ phases, exhibiting similar cluster trends. We observe that the Zener ratios (a dimensionless number used to quantify the anisotropy of cubic crystals; the larger the deviation from one, the stronger the anisotropy) of $\mathrm{Cr}$ and $\mathrm{V}$ are $<1$, whereas those of the other elements 

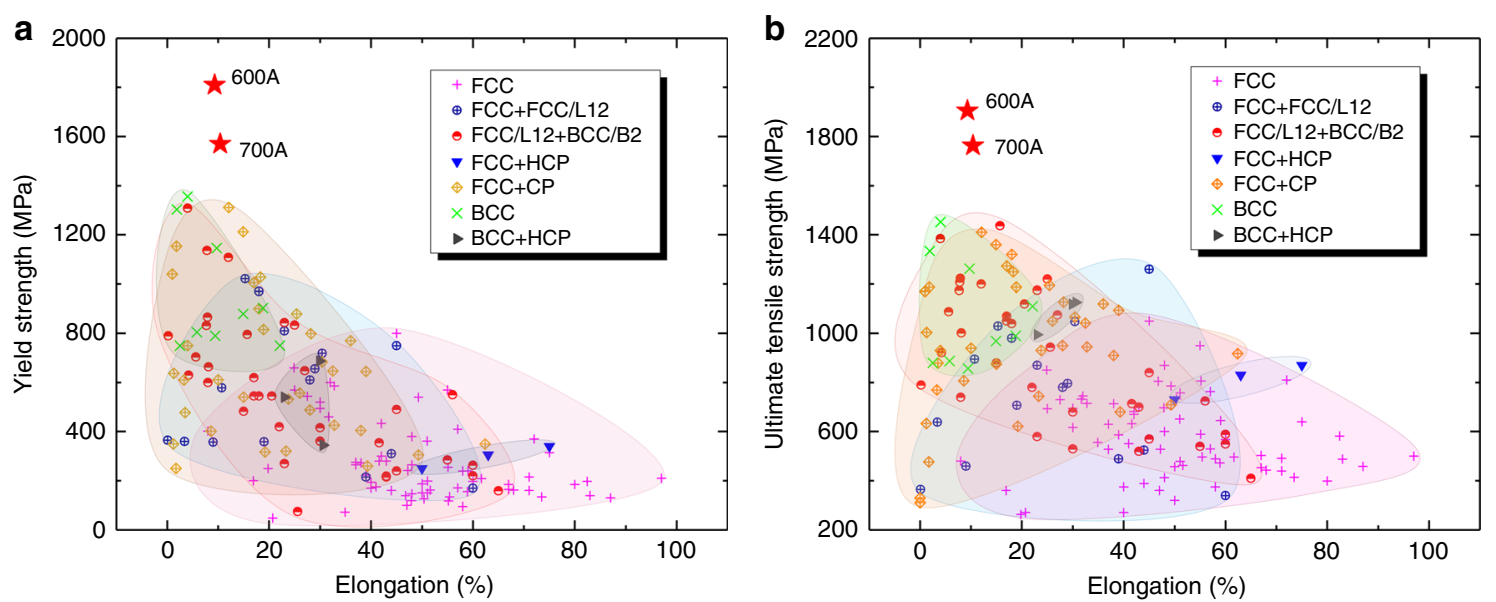

Fig. 4 Comparison of our developed HEAs (600 A and 700 A) with existing HEAs. a, b Maps of elongation versus YS and UTS of HEAs reported previously at room temperature, respectively, where HCP and CP refer to the phases with hexagonal close-packed and complex lattice structures (e.g. $\sigma$ and $\mu$ phases), respectively. The data of the tensile properties of reported HEAs were acquired from Supplementary Table 1

are greater than one, and $\mathrm{Cr}$ has the highest elastic parameters $^{21,27}$, suggesting that such a cluster can reduce the anisotropy of the matrix and stabilize this distinctive isotropic spinodal nanostructure. Since the spinodal nanostructure is isotropic, no preferred orientation exists during phase separation, leading to no additional satellite spots appearing in the SAED patterns (Fig. 2c, d). It should be noted that phase separation phenomena including spinodal decomposition are common in HEAs ${ }^{1,28-38}$. It is therefore that this work can utilize them to create a PH-HEA in situ with high-content $\mathrm{L}_{2}$ precipitates from a non-equiatomic system. More importantly, such a spinodal nanostructure should be closely related to the ultrahigh strength of our alloy because it contains ordered $\mathrm{L}_{2}$ nanoprecipitates with high density and volume fraction, which can introduce abundant low-misfit, coherent, nanoscale interfaces ${ }^{39}$ that can effectively strengthen metals, e.g. nanotwins ${ }^{39-41}$ and nanoprecipitates ${ }^{26}$.

In our HEAs, the lattice constant and modulus of the ordered $\mathrm{L}_{2}$ precipitates approximate to those of the disordered FCC matrix, and no new $\mathrm{FCC}-\mathrm{Ll}_{2}$ interfaces form after dislocations shear these $\mathrm{L}_{2}$ precipitates with diffuse coherent interfaces. Therefore, strengthening by coherent strain, modulus difference, and interfacial energy increase is negligible ${ }^{15-17}$. Instead, the $\mathrm{L}_{2}$ nanophases were formed by spinodal decomposition. This spinodal nanostructure can resist dislocation motion by providing strong diffuse attractive obstacles to trap moving dislocations and by creating $\mathrm{APBs}$ on the slip planes of these ordered $\mathrm{L}_{2}$ nanophases when they are sheared by dislocations ${ }^{19}$. The contribution of the spinodal nanostructure to the YS of $600 \mathrm{~A}$ is calculated to be $1158 \mathrm{MPa}$ (see Methods in details). Superposing additional strengthening from grain boundaries and the BCC phases, the total increase in YS is estimated to be $1524 \mathrm{MPa}$, which agrees with the experimental result of $1536 \mathrm{MPa}$, confirming that this spinodal order-disorder nanostructure can potently strengthen our alloys. Furthermore, no pile-up dislocations are found in the $\mathrm{FCC}+\mathrm{L}_{2}$ region (Supplementary Fig. 6), suggesting that the diffuse low-misfit coherent $\mathrm{FCC}-\mathrm{L}_{2}$ interfaces can minimize the elastic strain accumulation resulting from dislocation shear and hence prevent crack initiation at these interfaces, contributing to good ductility.

We compare the tensile properties of our PH-HEA with those of reported HEAs in Fig. 4. It is clear that the tensile properties depend on the phases present in alloys. FCC alloys are ductile but relatively soft, whereas BCC alloys are hard but relatively brittle, and strength-ductility combinations of multiphase alloys are generally better than those of single-phase ones. In particular, our alloys (600 A and $700 \mathrm{~A}$ ) have significant advantages over existing bulk HEAs, including the highest YS and UTS and a good strength-ductility balance, showing the enormous potential for advanced structural applications.

Our alloying strategy not only involves the change to the initial compositions (non-equiatomic), but also requires the formation of a near-equiatomic/high-entropy solid-solution matrix in situ after separating out enough strengthening phases. The latter requirement is the primary difference relative to the previous HEA design concepts, which only consider the initial compositions regardless of whether the final primary phases are highentropy solid solutions or not, leading to the possible loss of the HEA advantages. As a result, our alloying strategy can introduce desired strengthening phases with controllable contents while still possessing the advantages of HEAs, and the corresponding HEA has several unique advantages over previous reported HEAs, as well as other materials strengthened by abundant low-misfit coherent nanotwins ${ }^{39-41} /$ nanoprecipitates $^{26}$. First, our selected strengthening phases are ductile, highly coherent and low misfit with matrix (i.e. they can compatibly deform with the matrix), implying that increasing their content does not significantly degenerate the ductility. Controllable microstructure (by either alloying or processing design) provides numerous possibilities for the optimization of properties; for instance, the strength of our alloy can be controlled within a very wide range, at least, of $\sim 270-1900 \mathrm{MPa}$, and the largest elongation is $>40 \%$. Furthermore, benefiting from the advantages of the multi-principal-element/high-entropy matrix, high $\mathrm{Cr}$ addition can improve the resistance to corrosion and oxidation, and high $\mathrm{Al}$ content reduces the alloy density. These advantages present the potential for the application of our HEA to many rigorous service conditions, such as those subjected to very large loading and involving highly corrosive environment. More importantly, the spinodal nanostructure achieves an amazing strengthening effect $(>1.5$ $\mathrm{GPa})$, good ductility ( $>9 \%)$, and excellent work-hardening capacity (uniform elongation to fracture), which are higher than those induced by independent spherical hard nanoparticles reported recently $(\sim 1.1 \mathrm{GPa}, 8.2 \%$, and $3.8 \%$ uniform elongation, respectively $)^{26}$ because of the higher volume fractions and the different strengthening mechanisms. It should be noted that the exciting strengthening effect of the isotropic spinodal structure with ductile $\mathrm{Ni}_{3} \mathrm{Al}$-type $\mathrm{L}_{2}$ nanophases in our HEA is different from that of the anisotropic spinodal structures with NiAl-type B2 phases reported previously ${ }^{42,43}$. The tensile properties of these reported HEAs with the anisotropic spinodal structures were 
unavailable and the compressive properties were just reported. The strength data obtained from the two loading modes are inappropriate to be analogized. Many HEAs that have very high compressive strength are too brittle to bear tensile loading ${ }^{43-45}$, leading to inferior competitiveness compared with commercial alloys. These results imply that although the spinodal structures are common in HEAs, the strengthening mechanism of anisotropic spinodal structures is different from that of the isotropic spinodal structures in our HEA. The new strengthening mechanism of the isotropic spinodal nanostructure demonstrates another classical example of metallic strengthening by low-misfit coherent nanoscale interfaces/precipitates, revealing a new strategy to potently strengthen alloys without severely losing ductility. Overall, the distinctive microstructure and excellent mechanical properties of our HEAs present the possibility of development of new HEAs with ultrahigh strength, sufficient ductility, and resistance to corrosion and oxidation to compete with traditional structural materials.

\section{Methods}

Samples preparation. Master $\mathrm{Al}_{0.5} \mathrm{Cr}_{0.9} \mathrm{FeNi}_{2.5} \mathrm{~V}_{0.2}$ alloy ingots were prepared by arc-melting pure metals ( $\mathrm{Al}, \mathrm{Cr}, \mathrm{Fe}, \mathrm{Ni}$, and $\mathrm{V}$ with purity $>9.9 \%$ ) under highpurity argon atmosphere. During preparation, the ingots were inverted and remelted at least four times to improve chemical homogeneity. These master alloy ingots were subsequently casted into a copper mold with a dimension of $50 \times 13 \times$ $30 \mathrm{~mm}^{3}$. To reduce elemental segregation, the as-cast alloys were solution-treated at $1200^{\circ} \mathrm{C}$ for $24 \mathrm{~h}$ followed by water quenching. The solution-treated alloys were then cold-rolled in steps at room temperature with a total rolling reduction ratio of $72 \%$. Finally, the as-rolled samples were aged at 600 and $700^{\circ} \mathrm{C}$ for $1 \mathrm{~h}$ followed by air cooling.

Tensile testing. Flat, dog-bone-shaped tensile samples with a gauge length of 10 $\mathrm{mm}$, a width of $3 \mathrm{~mm}$ and a thickness of $1 \mathrm{~mm}$ were cut by electrical discharge machining, and polished with 2000 -grit $\mathrm{SiC}$ papers. Tensile tests were performed by a CMT4305 universal electronic tensile testing machine at room temperature using a nominal strain rate of $1 \times 10^{-3} \mathrm{~s}^{-1}$.

Microstructural characterization. Grain morphology was characterized by a Zeiss Axio Observer A1m inverted optical microscope (OM) and a JEOL JSM-7001F scanning electron microscope with a TSL OIM 6.2 system for electron backscatter diffraction (EBSD) analysis. The specimens were ground with 2000 -grit $\mathrm{SiC}$ papers, then mechanically polished using 2.5 - and 0.5 -grit diamond pastes, and finally polished using oxide polish suspension. The surfaces of the OM specimens were corroded by Marble's corrosive liquid to show grain boundaries. The EBSD scanning step is $0.05 \mu \mathrm{m}$, and the data are processed and analyzed by TSL OIM 6.2 software.

Phases existing in the current alloys were investigated by a synchrotron-based high-energy X-ray diffraction technique, at the 11-ID-C beam line of the Advanced Photon Source, Argonne National Laboratory, USA. The wavelength $\lambda$ of the high energy X-ray used here is $0.011725 \mathrm{~nm}$. To obtain precise lattice parameters $a$, we calculated the lattice parameters of all peaks in the HEXRD spectra, and plotted them versus $f(\theta)=\left(\cos ^{2} \theta / \sin \theta+\cos ^{2} \theta / \theta\right) / 2$, where $\theta$ is the Bragg angle of the diffraction peaks ${ }^{46}$. We then fitted the plot using the linear least squares method and extrapolated to $f(\theta)=0$, i.e. $\theta=90^{\circ}$, where the lattice parameter $a$ is the precise value. The results showed that although the lattice parameter of the ST sample is $3.5962 \pm 0.0006 \AA$, the lattice parameter of the FCC matrix of $600 \mathrm{~A}(3.5815 \pm$ $0.0008 \AA$ ) with a high $\mathrm{Cr}$ content was decreased to a value very close to that of the $\mathrm{L}_{2}$ precipitates $(3.5832 \pm 0.0009 \AA)$. The \pm values mean the standard deviation given by the linear least squares fitting. The mean size (particle diameter $d$ ) of the ordered $\mathrm{L}_{2}$ precipitates was determined according to the Scherrer equation $d=$ $K \lambda / \beta \cos \theta^{47}$, where $\beta$ is the full width at half maximum (FWHM) of the diffraction peak (in radian) and $K$ is a constant and approximates to 0.9 for cubic symmetry ${ }^{48}$. Many factors such as fine particle size, chemical heterogeneities, stress gradients, and microstresses can cause broadening of the (100) superlattice peak of the $\mathrm{Ll}_{2}$ phases. Fortunately, in addition to fine particle size, other factors broaden both the superlattice and fundamental peaks. The difference between the FWHM of the (100) superlattice peak $\left(\sim 0.043^{\circ}\right)$ and that of the fundamental peaks $\left(\sim 0.026^{\circ}\right)$ in $600 \mathrm{~A}$ is the broadening resulting from the fine particle size ${ }^{49}$, affording a mean diameter of the $\mathrm{L}_{2}$ phases of $\sim 35 \mathrm{~nm}$ in $600 \mathrm{~A}$. The highest peaks of $\mathrm{FCC}+\mathrm{Ll}_{2}$ (111) and BCC (110) are clearly overlapped. To reduce error, we performed the quantitative phase analysis based on the secondary high peak of each phase, i.e. the (200) fundamental peak of the FCC $+\mathrm{Ll}_{2}$, the (100) superlattice peak of the $\mathrm{Ll}_{2}$, and the (211) peak of the BCC. Another difficulty is lied in distinguishing the contributions of FCC and $\mathrm{L}_{2}$ to the (200) fundamental peak. Here we assume that (1) the $\mathrm{L}_{2}$ phases is of perfect order, and (2) the contributions of the FCC and $\mathrm{L}_{2}$ to the peak intensity depend on only their contents. Marking FCC, $\mathrm{L}_{2}$, and BCC as subscripts 1,2 , and 3 , respectively, there is:

$$
\left\{\begin{array}{c}
\frac{I^{100}}{I^{200}}=\frac{C_{2}^{100} f_{2}}{C_{1+2}^{200} f_{1}+C_{2}^{200} f_{2}} \\
\frac{I_{3}^{211}}{I_{2}^{100}}=\frac{C_{3}^{211} f_{3}}{C_{2}^{100} f_{2}} \\
\sum_{j=1}^{3} f_{j}=1
\end{array}\right.
$$

where $I_{i j}$ is the intensity of the $i$ peak for the $j$ phase, $f_{j}$ is the fraction volume of the $j$ phase, and $C_{i}$ is a parameter related to phase composition, Bragg angle, lattice structure, and phase nature. For synchrotron source, the absorptivity and Bragg angle are rather small, and hence $C_{i j}$ can be evaluated according to the APT result. The volume fractions of the FCC, $\mathrm{Li}_{2}$, and BCC phases in $600 \mathrm{~A}$ were calculated to be $43.7 \%, 50.1 \%$, and $6.2 \%$, respectively. It should be noted that these values may not be absolutely precise, but they can serve as reasonable references for analysis of the strengthening mechanism in our ultrastrong PH-HEAs.

Detailed microstructure was characterized by a JEOL ARM200F sphericalaberration corrected transmission electron microscope (TEM), and atomicresolution images were taken by a high-angle annular dark-field (HAADF) detector. The TEM specimens with dimensions of $\Phi 3 \mathrm{~mm} \times 0.5 \mathrm{~mm}$ were cut with a diamond wire saw and thinned to $40-60 \mu \mathrm{m}$ by 3000 - and 5000 -grit SiC papers. After mechanically polishing, twin-jet electro-polishing was conducted using a mixed solution of $\mathrm{HNO}_{3}: \mathrm{CH}_{3} \mathrm{OH}=1: 4$ at a temperature of $\sim 235 \mathrm{~K}$ with a direct voltage of $28 \mathrm{~V}$ and a current of $55 \mathrm{~mA}$. Final polishing was conducted on a Gatan 691 precision ion polishing system using a voltage of $2 \mathrm{kV}$ for $7.5 \mathrm{~min}$ and a voltage of $1 \mathrm{kV}$ for $15 \mathrm{~min}$.

Atom probe tomography (APT) analysis for three-dimensional distribution of elements in materials was performed by a CAMECA local electrode atom probe LEAP $^{\mathrm{TM}} 4000 \mathrm{X}$ SI at a target specimen temperature of $20 \mathrm{~K}$, under a pulsing UV laser with a pulse energy of $40 \mathrm{pJ}$, a pulse rate of $200 \mathrm{kHz}$, and an ion collection rate of $0.5 \%$ per pulse. A two-stage electro-polishing procedure was used to fabricate a sharp tip specimen (apex radius $<50 \mathrm{~nm}$ ) for APT analyses. The first stage electropolishing was performed using an electrolyte containing 25 volume per cent (vol. $\%)$ perchloric acid in acetic acid at $15 \mathrm{~V}$ and room temperature, and the second stage was by a 2 vol.\% perchloric acid in 2-butoxyethanol solution at $20 \mathrm{~V}$. Reconstruction and quantitative analysis of the APT data were conducted on a CAMECA IVAS version 3.6.8 software. The compositions of the FCC, $\mathrm{L}_{2}$ and $\mathrm{BCC}$ phases were estimated by averaging the core concentrations of the proximity histograms produced by reconstructing $22 \% \mathrm{Cr}, 63 \% \mathrm{Ni}$, and $50 \% \mathrm{Cr}$ (in at $\%$ ) isoconcentration surfaces, respectively.

Estimation of strengthening by various mechanisms. For near-equiatomic HEAs, strengthening by solute atoms is difficult to evaluate because distinguishing between the solvent and solute is difficult. Fortunately, solution strengthening should be similar for a given system, and hence the difference in the strength of ST alloys is attributed to various grain sizes. The intercept term $\sigma_{0}$ in the Hall-Petch equation $\left(\Delta \sigma_{\mathrm{gs}}=\sigma_{0}+k_{\mathrm{g}} \cdot d^{-0.5}\right.$, where $k_{\mathrm{g}}$ is the Hall-Petch coefficient and $d$ is the mean grain size $)^{21}$ can be considered an alloy constant composed of solution strengthening $\Delta \sigma_{\mathrm{ss}}$ and lattice friction stress $\sigma_{\mathrm{fs}}$. This consideration can reasonably simplify the estimation of the strengthening effect in multi-principal-element HEAs. To obtain different grain sizes, we annealed the as-rolled samples at $1200^{\circ} \mathrm{C}$ for various hold times $(10,30$, and $120 \mathrm{~min})$, and measured the grain sizes and tensile properties of these as-annealed samples. Using Hall-Petch equation to fit the experimental results (Supplementary Fig. 2), $\sigma_{0}$ and $k_{\mathrm{g}}$ were determined to be $270.0 \mathrm{MPa}$ and $378.6 \mathrm{MPa} \cdot \mu \mathrm{m}^{0.5}$, respectively, where $\sigma_{0}=\sigma_{\mathrm{fs}}+\Delta \sigma_{\mathrm{ss}}$. The mean grain sizes of $600 \mathrm{~A}$ and $700 \mathrm{~A}$ are $2.82 \pm 0.77$ and $1.62 \pm 0.53 \mu \mathrm{m}$, respectively. The \pm values mean the standard deviation given by EBSD. The contributions of grain boundaries to the yield strengths of the two samples are 212 and $284 \mathrm{MPa}$, respectively. Since recovery and recrystallization have been completed in all the samples after the final heat treatment, dislocation strengthening is negligible.

For spinodal structure, dislocations will be trapped by strong diffuse obstacles. The equation for evaluation of strengthening by spinodal structure is ${ }^{19}$ :

$$
\Delta \sigma_{\mathrm{sds}}=M k_{\mathrm{s}}(A \eta Y)^{5 / 3}(\lambda / G b)^{2 / 3}
$$

where $M=3.06$ is the Taylor factor, $k_{\mathrm{s}}=0.122 \cdot[\pi(1-v) /(1-2 v)]^{2 / 3}$ is a material constant, $v=0.31$ is the Poisson ratio, $A=\left(C-C_{0}\right) / 3$ is the amplitude of that modulation (in at\%), $\lambda$ is the wavelength of the composition modulation, and $\eta=a^{-1} \cdot d a / d C$ is the lattice strains produced by spinodal decomposition, in which $a$ is the lattice constant for the undecomposed sample, $d a / d C$ is the variation in the lattice constant with the composition $C$. According to the HEXRD data, the lattice constants of the undecomposed ST sample and FCC matrix in 600 A are 0.3596 and $0.3582 \mathrm{~nm}$, respectively. Since the current system (600 A sample) is isotropic, $E=162 \mathrm{GPa}$ is the elastic modulus, $Y=E /(1-v)=234.8 \mathrm{GPa}$ is an elasticdependent parameter, $G=61.8 \mathrm{GPa}$ is the shear modulus, and $b=\sqrt{2} a / 2$ is the Burgers vector. According to Supplementary Fig. $4 \mathrm{~b}, \lambda$ is evaluated to be $\sim 40 \mathrm{~nm}$, whereas this value approximates to $70 \mathrm{~nm}(2 d)$ based on the HEXRD result. However, the value evaluated based on the APT data is quite local while that 
calculated by the Scherrer equation is based on an assumption of spherical particles. Therefore, we use a $\lambda$ value of $60 \mathrm{~nm}$ for calculation. In the current multiprincipal-element system, it is difficult to evaluate the value of $A$. We observe that $\mathrm{Fe}$ and $\mathrm{Cr}$ exhibit similar cluster trends, and the content of $\mathrm{Ni}+\mathrm{Al}+\mathrm{V}$ is greater than 60 at $\%$ according to the APT data. We therefore assume that $\mathrm{Ni}+\mathrm{Al}+\mathrm{V}$ is the solvent for simplification. After ageing $1 \mathrm{~h}$ at $600^{\circ} \mathrm{C}$, the solute concentrations change from 39.34 at\% in the ST sample to 54.57 at\% in the FCC matrix (rich region) based on the APT data. Accordingly, $A=5.1$ at $\%$ and $\Delta \sigma_{\mathrm{sds}}$ is calculated to be $406 \mathrm{MPa}$.

Strengthening by ordered coherent precipitates $\Delta \sigma_{\text {os }}$ occurs when dislocations shear these ordered phases and create APBs on the slip planes of them. We find that the distances between two coupled dislocations $(\sim 20-40 \mathrm{~nm}$, as shown in Supplementary Fig. 6d) approximate to the mean diameter of the ordered $\mathrm{L}_{2}$ phases $(\sim 30 \mathrm{~nm})$, suggesting that the coupling of the dislocation in the pairs should be moderate (between strong and weak), and that the alloy should be aged to near the peak strength condition. Therefore, the YS increase $\Delta \sigma_{\text {os }}$ is given by ${ }^{19}$ :

$$
\Delta \sigma_{\mathrm{os}}=M 0.81 \frac{\gamma_{\mathrm{APB}}}{2 b}\left(\frac{3 \pi f}{8}\right)^{1 / 2}
$$

in which $f$ is the volume fraction of the ordered precipitates, $r$ is the average radius of these ordered phases, and $\gamma_{\mathrm{APB}}=0.2 \mathrm{~J} \mathrm{~m}^{-2}$ is the APB energy that is adopted from the data of $\mathrm{Ni}_{3} \mathrm{Al}$ precipitates ${ }^{50}$. Assuming that the $\mathrm{L1}_{2}$ precipitates are near spherical, $f \approx 50 \%$ and $r$ is assumed to be $1 / 4 \lambda(\sim 15 \mathrm{~nm})$ in $600 \mathrm{~A}$; hence $\Delta \sigma_{\text {os }}$ is calculated to be $752 \mathrm{MPa}$. It should be noted that in our PH-HEAs the ordered $\mathrm{Ll}_{2}$ nanoprecipitates are irregular and interconnected rather than spherical. The increases in YS calculated based on independent spheres is therefore not absolutely accurate and may underestimate the area of APBs and the strengthening effect. Nevertheless, the results can offer reasonable references to analyze the strengthening caused by the current spinodal order-disorder nanostructure.

Considering the $\mathrm{BCC}$ phases as the precipitates within $\mathrm{FCC}+\mathrm{Ll}_{2}$ matrix, we find that dislocations can shear and pass through these precipitates (Supplementary Fig. $6 \mathrm{a}-\mathrm{c})$. It is noteworthy that two orientation relationships, i.e. $[\overline{1} 11]_{\mathrm{BCC}} / /[011]_{\mathrm{FCC}}$ and $(110)_{\mathrm{BCC}} / /(11 \overline{1})_{\mathrm{FCC}}$, exist between the BCC phases and matrix (Supplementary Fig. 7). These observations mean that these BCC phases share the slide planes with the matrix, and that the specific energy of new interfaces created by shearing is low, leading to weak chemical strengthening. Moreover, elastic lattice strain induced by semi-coherent precipitate-matrix interfaces is low enough to be negligible. Instead, the difference in shear moduli between the BCC precipitates and matrix is high $\left(\Delta G=G_{\mathrm{BCC}}-G_{\text {matrix }}=100-85=15 \mathrm{GPa}\right.$, where $G_{\mathrm{BCC}}$ is adopted from the data of a $\mathrm{Cr}-22$ at $\% \mathrm{Fe}$ alloy ${ }^{51,52}$ and $G_{\text {matrix }}$ is a value selected between the shear modulus of $\mathrm{CoCrFeNi}^{17}$ and that of $\mathrm{CoCrNi}^{16}$ ). Therefore, modulus strengthening $\Delta \sigma_{\mathrm{ms}}$, which should be the primary contribution to the strength increase, is given by ${ }^{19}$ :

$$
\Delta \sigma_{\mathrm{ms}}=M 0.0055(\Delta G)^{\frac{3}{2}}\left(\frac{2 f}{G}\right)^{\frac{1}{2}}\left(\frac{r}{b}\right)^{\frac{3 m}{2}-1}
$$

where $m$ is a constant and is equal to 0.85 for FCC-based alloys. In the $600 \mathrm{~A}$ sample, the BCC phases are plate-shaped with dimensions of $\sim 500 \times 50 \times 10 \mathrm{~nm}^{3}$. For simplification, we assume the BCC phases to be spherical with diameters of 50 $\mathrm{nm}$ because the projected lengths of the BCC phases at the slide planes approximate to this value. Accordingly, $\Delta \sigma_{\mathrm{ms}}$ is calculated to be $154 \mathrm{MPa}$. It is worth pointing out that this assumption significantly increases the number density of the BCC phases for a fixed $f$, leading to overestimation of the contribution of the BCC phases to the yield strength. Nevertheless, strengthening by the BCC precipitates is confirmed to be low, and the calculated result agrees well with the actual increases in strength.

\section{Data availability}

The data that support the findings of this study are available from the corresponding author on reasonable request.

Received: 14 July 2018 Accepted: 14 September 2018

Published online: 03 October 2018

\section{References}

1. Yeh, J.-W. et al. Nanostructured high-entropy alloys with multiple principal element: novel alloy design concepts and outcomes. Adv. Eng. Mater. 6, 299-303 (2004).

2. Zhang, Y. et al. Microstructures and properties of high-entropy alloys. Prog. Mater. Sci. 61, 1-93 (2014).

3. Pickering, E. J. \& Jones, N. G. High-entropy alloys: a critical assessment of their founding principles and future prospects. Int. Mater. Rev. 61, 183-202 (2016).
4. Gludovatz, B. et al. A fracture-resistant high-entropy alloy for cryogenic applications. Science 345, 1153-1158 (2014).

5. Liu, W. H., Yang, T. \& Liu, C. T. Precipitation hardening in CoCrFeNi-based high entropy alloys. Mater. Chem. Phys. 210, 2-11 (2018).

6. Miracle, D. B. \& Senkov, O. N. A critical review of high entropy alloys and related concepts. Acta Mater. 122, 448-511 (2016).

7. Liu, W. H. et al. Ductile CoCrFeNiMox high entropy alloys strengthened by hard intermetallic phases. Acta Mater. 116, 332-342 (2016).

8. Ming, K., Bi, X. \& Wang, J. Precipitation strengthening of ductile $\mathrm{Cr}_{15} \mathrm{Fe}_{20} \mathrm{Co}_{35} \mathrm{Ni}_{20} \mathrm{Mo}_{10}$ alloys. Scr. Mater. 137, 88-93 (2017).

9. Wani, I. S. et al. Tailoring nanostructures and mechanical properties of $\mathrm{AlCoCrFeNi}_{2.1}$ eutectic high entropy alloy using thermo-mechanical processing. Mater. Sci. Eng. A 675, 99-109 (2016).

10. Li, Z., Pradeep, K. G., Deng, Y., Raabe, D. \& Tasan, C. C. Metastable highentropy dual-phase alloys overcome the strength-ductility trade-off. Nature 534, 227-230 (2016).

11. Lu, Y. et al. Directly cast bulk eutectic and near-eutectic high entropy alloys with balanced strength and ductility in a wide temperature range. Acta Mater. 124, 143-150 (2017).

12. Huang, $H$. et al. Phase-transformation ductilization of brittle high-entropy alloys via metastability engineering. Adv. Mater. 29, 1701678 (2017).

13. $\mathrm{Li}$, D. et al. High-entropy $\mathrm{Al}_{0.3} \mathrm{CoCrFeNi}$ alloy fibers with high tensile strength and ductility at ambient and cryogenic temperatures. Acta Mater. 123, 285-294 (2017).

14. Gwalani, B. et al. Optimizing the coupled effects of Hall-Petch and precipitation strengthening in a $\mathrm{Al}_{0.3} \mathrm{CoCrFeNi}$ high entropy alloy. Mater. Des. 121, 254-260 (2017).

15. Wang, Z. G. et al. Effect of coherent $\mathrm{L}_{2}$ nanoprecipitates on the tensile behavior of a fcc-based high-entropy alloy. Mater. Sci. Eng. A 696, 503-510 (2017).

16. Zhao, Y. L. et al. Heterogeneous precipitation behavior and stacking-faultmediated deformation in a CoCrNi-based medium-entropy alloy. Acta Mater. 138, 72-82 (2017)

17. He, J. Y. et al. A precipitation-hardened high-entropy alloy with outstanding tensile properties. Acta Mater. 102, 187-196 (2016).

18. Liu, C. T. \& Stiegler, J. O. Ductile ordered intermetallic alloys. Science 226, 636-642 (1984).

19. Ardell, A. J. Precipitation hardening. Metall. Trans. A 16A, 2131-2165 (1985).

20. Reed, R. C. The Superalloys: Fundamentals and Applications. (Cambridge university press, New York, 2006).

21. Courtney, T. H. Mechanical Behavior of Materials. Second edn. (McGraw-Hill, Boston, 2000)

22. Cahn, J. W. Phase separation by spinodal decomposition in isotropic systems. J. Chem. Phys. 42, 93-99 (1965).

23. Viswanathan, G. B. et al. Precipitation of ordered phases in metallic solid solutions: a synergistic clustering and ordering process. Scr. Mater. $\mathbf{6 5}$, 485-488 (2011).

24. Booth-Morrison, C. et al. Effects of solute concentrations on kinetic pathways in Ni-Al-Cr alloys. Acta Mater. 56, 3422-3438 (2008).

25. $\mathrm{Lu}, \mathrm{K}$. Stabilizing nanostructures in metals using grain and twin boundary architectures. Nat. Rev. Mater. 1, 16019 (2016).

26. Jiang, S. et al. Ultrastrong steel via minimal lattice misfit and high-density nanoprecipitation. Nature 544, 460-464 (2017).

27. Lenkkeri, J. T. \& Lahteenkorva, E. E. An investigation of elastic moduli of vanadium-chromium alloys. J. Phys. F Metal Phys. 8, 1643-1651 (1978).

28. Singh, S., Wanderka, N., Murty, B. S., Glatzel, U. \& Banhart, J. Decomposition in multi-component AlCoCrCuFeNi high-entropy alloy. Acta Mater. 59, 182-190 (2011).

29. Santodonato, L. J. et al. Deviation from high-entropy configurations in the atomic distributions of a multi-principal-element alloy. Nat. Commun. 6, 5964 (2015).

30. Otto, F. et al. Decomposition of the single-phase high-entropy alloy $\mathrm{CrMnFeCoNi}$ after prolonged anneals at intermediate temperatures. Acta Mater. 112, 40-52 (2016).

31. Wen, L. H. et al. Effect of aging temperature on microstructure and properties of AlCoCrCuFeNi high-entropy alloy. Intermetallics 17, 266-269 (2009).

32. Singh, S., Wanderka, N., Kiefer, K., Siemensmeyer, K. \& Banhart, J. Effect of decomposition of the $\mathrm{Cr}-\mathrm{Fe}-\mathrm{Co}$ rich phase of $\mathrm{AlCoCrCuFeNi}$ high entropy alloy on magnetic properties. Ultramicroscopy 111, 619-622 (2011).

33. Wang, W.-R. et al. Effects of $\mathrm{Al}$ addition on the microstructure and mechanical property of $\mathrm{Al}_{\mathrm{X}} \mathrm{CoCrFeNi}$ high-entropy alloys. Intermetallics $\mathbf{2 6}$, 44-51 (2012).

34. Pickering, E. J., Stone, H. J. \& Jones, N. G. Fine-scale precipitation in the highentropy alloy $\mathrm{Al}_{0.5} \mathrm{CrFeCoNiCu}$. Mater. Sci. Eng. A 645, 65-71 (2015).

35. Tong, C.-J. et al. Mechanical performance of the $\mathrm{Al}_{\mathrm{X}} \mathrm{CoCrCuFeNi}$ highentropy alloy system with multiprincipal elements. Metall. Mater. Trans. A 36A, 1263-1271 (2005) 
36. Tong, C.-J. et al. Microstructure characterization of $\mathrm{Al}_{\mathrm{X}} \mathrm{CoCrCuFeNi}$ highentropy alloy system with multiprincipal elements. Metall. Mater. Trans. A 36A, 881-893 (2005).

37. Tung, C.-C. et al. On the elemental effect of AlCoCrCuFeNi high-entropy alloy system. Mater. Lett. 61, 1-5 (2007).

38. Manzoni, A., Daoud, H., Volkl, R., Glatzel, U. \& Wanderka, N. Phase separation in equiatomic AlCoCrFeNi high-entropy alloy. Ultramicroscopy 132, 212-215 (2013).

39. Lu, K., Lu, L. \& Suresh, S. Strengthening materials by engineering coherent internal boundaries at the nanoscale. Science 324, 349-352 (2009).

40. Lu, L., Chen, X., Huang, X. \& Lu, K. Revealing the maximum strength in nanotwinned copper. Science 323, 607-610 (2009).

41. Li, X., Wei, Y., Lu, L., Lu, K. \& Gao, H. Dislocation nucleation governed softening and maximum strength in nano-twinned metals. Nature 464, 877-880 (2010).

42. Hanna, J. A., Baker, I., Wittmann, M. W. \& Munroe, P. R. A new highstrength spinodal alloy. J. Mater. Res. 20, 791-795 (2011).

43. Xia, S., Yang, X., Chen, M., Yang, T. \& Zhang, Y. The Al effects of Co-free and V-containing high-entropy alloys. Metals 7, 18 (2017).

44. Liu, S., Gao, M. C., Liaw, P. K. \& Zhang, Y. Microstructures and mechanical properties of $\mathrm{Al}_{\mathrm{X}} \mathrm{CrFeNiTi}_{0.25}$ alloys. J. Alloy Compd. 619, 610-615 (2015).

45. Laktionova, M. A., Tabchnikova, E. D., Tang, Z. \& Liaw, P. K. Mechanical properties of the high-entropy alloy $\mathrm{Ag}_{0.5} \mathrm{CoCrCuFeNi}$ at temperatures of 4.2-300 K. Low Temp. Phys. 39, 630-632 (2013).

46. Nelson, J. B. \& Riley, D. P. An experimental investigation of extrapolation methods in the derivation of accurate unit-cell dimensions of crystals. Proc Phys. Soc. 57, 160-177 (1945).

47. Patterson, A. L. The scherrer formula for X-ray particle size determination. Phys. Rev. 56, 978-982 (1939).

48. Langford, J. I. \& Wilson, A. J. C. Seherrer after sixty years: a survey and some new results in the determination of crystallite size. J. Appl. Crystallogr. 11, 102-113 (1978).

49. Ungár, T. Microstructural parameters from X-ray diffraction peak broadening. Scr. Mater. 51, 777-781 (2004).

50. Kozar, R. W. et al. Strengthening mechanisms in polycrystalline multimodal nickel-base superalloys. Metall. Mater. Trans. A 40, 1588-1603 (2009).

51. Lenkkeri, J. T. The elastic moduli of some body-centred cubic titaniumvanadium, vanadium-chromium and chromium-iron alloys. J. Phys. F Metal. Phys. 10, 611-618 (1980).

52. Razumovskiy, V. I., Ruban, A. V. \& Korzhavyi, P. A. First-principles study of elastic properties of Cr- and Fe-rich Fe-Cr alloys. Phys. Rev. B 84, 024106 (2011).

\section{Acknowledgements}

Y.X. acknowledges support by the National Natural Science Foundation of China (NSFC) (grant numbers 51471035 and 51701018). Y.J.L. acknowledges the Project Funded by
China Postdoctoral Science Foundation (grant number 2017M620019). X.L. acknowledges support by the NSFC (grant numbers 11522218 and 51420105001). Use of the Advanced Photon Source is supported by the U.S. Department of Energy, Office of Science, Office of Basic Energy Sciences, under Contract No. DE-AC02-06CH11357. We thank Dr. F.-K. Chiang at National Institute of Clean-and-Low-Carbon Energy, China for help in the STEM experiments, thank Dr. S.B. Jin at the Materials Characterization Facility of Nanjing University of Science and Technology for help in the APT experiments, and thank Prof. Z.H. Nie at Beijing Institute of Technology for help in the HEXRD analysis.

\section{Author contributions}

Y.X., F.W., X.L., and Y.J.L. designed the study. Y.J.L., L.j.W., Y.r.W., Y.X., and X.L. analyzed the data and wrote the paper. L.j.W., B.C., T.C., and Q.X. carried out the main experiments. Y.r.W. conducted the STEM characterization and interpreted the results. Q. W. and G.S. conducted the APT experiments and interpreted the results. Y.W. and Y.R conducted the synchrotron experiments and analyzed the results. L.W., F.W., and H.C. reconfirmed the mechanical properties. All authors contributed to the discussion of the results, and commented on the manuscript.

\section{Additional information}

Supplementary Information accompanies this paper at https://doi.org/10.1038/s41467018-06600-8.

Competing interests: The authors declare no competing interests.

Reprints and permission information is available online at http://npg.nature.com/ reprintsandpermissions/

Publisher's note: Springer Nature remains neutral with regard to jurisdictional claims in published maps and institutional affiliations.

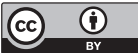

Open Access This article is licensed under a Creative Commons Attribution 4.0 International License, which permits use, sharing, adaptation, distribution and reproduction in any medium or format, as long as you give appropriate credit to the original author(s) and the source, provide a link to the Creative Commons license, and indicate if changes were made. The images or other third party material in this article are included in the article's Creative Commons license, unless indicated otherwise in a credit line to the material. If material is not included in the article's Creative Commons license and your intended use is not permitted by statutory regulation or exceeds the permitted use, you will need to obtain permission directly from the copyright holder. To view a copy of this license, visit http://creativecommons.org/ licenses/by/4.0/

(C) The Author(s) 2018 